\title{
Creatividad y desarrollo cognitivo en personas mayores
}

\section{Creativity and cognitive development in older people}

\author{
Silvia Carrascal \\ Facultad de Educación. Universidad Internacional de la Rioja. \\ Facultad de Ciencias Sociales y de la Educación. Universidad Camilo José Cela. \\ Escuela Universitaria - ESCUNI. Universidad Complutense de Madrid. \\ silvia.carrascal@unir.net \\ Eva Solera \\ Facultad de Educación. Universidad Internacional de la Rioja. \\ eva.solera@unir.net
}

Recibido: 20 de septiembre de 2012

Aprobado: 23 de febrero de 2013

\section{Resumen}

El progresivo envejecimiento de la población ha provocado el desarrollo de múltiples formas de intervención para mejorar su calidad de vida. En diversas investigaciones sobre el tema se ha demostrado que a través de estas estrategias se consigue paliar o frenar el deterioro cognitivo en cierta medida.

A pesar de la dependencia física y psíquica que pueden tener, conservan los rasgos específicos inherentes en la persona: singularidad, autonomía y voluntad. Podemos decir que el hombre no solo vive, sino que convive; por lo que se hace necesario su relación y comunicación con otros.

El uso didáctico de la imagen y las artes plásticas visuales para el desarrollo y motivación de la creatividad, analizando su contribución al desarrollo cognitivo, las habilidades y las destrezas de las personas mayores, favorecerá su desarrollo personal.

En este artículo analizamos la importancia del desarrollo de la creatividad en la vida cotidiana de los mayores, tanto de forma activa como pasiva, para mantenerles en contacto con el entorno y conservar sus capacidades cognitivas, afectivas y sociales. Además proponemos diferentes actividades para favorecer el desarrollo cognitivo a través de la estimulación creativa buscando el equilibrio entre los conocimientos cognitivos y emocionales y atendiendo a lo más inherente del ser humano: su capacidad de adaptarse.

Palabras clave: desarrollo cognitivo, estimulación cognitiva, inteligencia emocional, artes y creatividad. 
Carrascal, S., Solera, E. (2013): Creatividad y desarrollo cognitivo en personas mayores. Arte, Individuo y Sociedad, 26 (1) 9-19

\begin{abstract}
The progressive aging of the population has induced to the development of multiple forms of intervention to improve their quality of life. In several studies on the subject has shown that through these strategies is achieved alleviate or slow the cognitive decline somewhat.

Despite the physical and psychological dependence can have, retain the specific features inherent in the person: uniqueness, autonomy and will. We can say that the man not only lives, but lives, so it is necessary to their relationship and communication with others.

The educational use of the image and the visual arts to the development and encouragement of creativity, analyzing their contribution to cognitive abilities and skills of older people, and foster their personal development.

In this paper we analyze the importance of developing creativity in everyday life of older people, both active and passive, to keep in contact with the environment and maintain their cognitive, emotional and social. We also propose different activities to promote cognitive development through creative stimulation seeking a balance between cognitive and emotional knowledge and serving as inherent human beings: their capacity to adapt.
\end{abstract}

Key words: cognitive development, Cognitive stimulation, Emotional intelligence, arts and creativity.

Carrascal, S., Solera, E. (2013): Creativity and cognitive development in older people. Arte, Individuo y Sociedad, 26 (1) 9-19

Sumario: 1. Introducción, 2. Desarrollo cognitivo en personas mayores, 2.1. Evolución y desarrollo cognitivo en personas mayores, 2.2. Estimulación cognitiva y aprendizaje, 2.2.1 Necesidad de estimulación cognitiva y aprendizaje, 2.2.2. Estimulación cognitiva: propuestas de intervención, 3. Creatividad y desarrollo cognitivo, 3.1. Motivación y desarrollo de habilidades cognitivas y creativas, 4. Conclusiones. Referencias.

\title{
1. Introducción
}

El progresivo envejecimiento de la población, unido al aumento de la esperanza de vida, ha dado lugar a la proliferación de numerosas investigaciones encaminadas a mejorar la atención a los mayores y su calidad de vida. El objetivo es conseguir que sus capacidades estén en el nivel más alto posible y lograr que sean personas activas, implicadas en la sociedad.

Aunque las habilidades creativas cobran especial relevancia en edades tempranas, el uso y motivación de la creatividad para favorecer el potencial creativo y el desarrollo de personas mayores, les permite adaptarse a su entorno con facilidad. Por ello, un programa de actividades artísticas y creativas que partan de la observación e interpretación libre de objetos, sería una herramienta capaz de mejorar los cambios evolutivos a nivel cognitivo, social y afectivo de las personas mayores. Su incapacidad no debe influir en su existencia.

En la evolución humana, el entorno físico y personal, resultan necesarios para el desarrollo y la subsistencia. Por ello, si el vínculo que pueda establecerse entre ambos, se ve deteriorado, resulta necesario el desarrollo de programas educativos que atiendan a esta diversidad cognitiva. 
Si fomentamos el desarrollo de habilidades cognitivas y afectivas mediante una planificación didáctica de actividades creativas, les permitiremos establecer relaciones entre los objetos de su entorno.

En este sentido, las artes plásticas visuales permitirán establecer esta relación necesaria entre la dimensión cognitiva y afectiva de las personas mayores, lo cual les permitirá recordar hechos, términos y conceptos básicos aprendidos con anterioridad.

Partiendo de dicha premisa veremos, a continuación, diferentes propuestas de intervención relacionadas con la motivación y el desarrollo de la creatividad.

\section{Desarrollo cognitivo en personas mayores}

El número de personas mayores de 65 años ha crecido en los últimos años y continuará incrementándose en el futuro (European Comission, 2012). Los datos indican que este grupo de edad representará el 30\% del total poblacional en 2060 (European Comission, 2012) y, ya en 2050, un $20 \%$ de estas personas tendrá más de 80 años (Naciones Unidas, 2009).

Esta situación, que ya se vislumbraba hace algunos años, ha dado lugar a múltiples estudios orientados a favorecer un envejecimiento saludable que permita prevenir enfermedades y deterioros físicos y psíquicos. Se trata de un interés completamente lógico puesto que tradicionalmente se ha asociado la vejez con la enfermedad (Fairwearther, 1991). Sin embargo, la gerontología también tiene en cuenta las diferencias individuales y analiza la forma de conseguir un envejecimiento óptimo o saludable (Fernández-Ballesteros, Caprara y García, 2005; López, 2008; Portal Mayores, 2006).

Esta idea ha llevado a numerosos investigadores a plantearse la importancia de desarrollar una serie de actividades para dar respuesta a las necesidades de este grupo poblacional, previniendo la aparición de déficits de todo tipo y proponiendo intervenciones adecuadas cuando la prevención no es posible (Albarracín y Berdullas, 2007).

A medida que la población mayor de 65 años ha ido aumentando y ha crecido su esperanza de vida hasta edades que no se habían alcanzado en épocas pasadas, las investigaciones han ido enfocando sus actuaciones hacia la mejora de la calidad de vida de este grupo de personas (Ballesteros, 2007; Díaz, Martín y Peraita, 2006; Portal Mayores, 2006; Tardif y Simard, 2011).

\subsection{Evolución y desarrollo cognitivo en personas mayores}

Actualmente los estudios gerontológicos tienen como objetivo fundamental conseguir un "envejecimiento activo", es decir, mantener al anciano activo física, mental y socialmente (Fernández-Ballesteros, 2004; Zamarrón, 2007). Esto implica desarrollar intervenciones o entrenamientos apropiados que motiven a la persona para involucrarse en la realización de actividades que incluyan procesos físicos, psíquicos y de interacción social. De esta manera el individuo se mantiene activo a todos los niveles y esto repercute en una buena salud y calidad de vida (López, 2008).

Anteriormente se asociaba la vejez con el declive físico y mental, parecía que nada se podía hacer para evitarlo o compensarlo. Sin embargo, desde hace décadas 
se habla de la plasticidad cerebral durante el envejecimiento, la capacidad de las personas mayores para desarrollar nuevos aprendizajes a partir de ciertos cambios neuronales que pueden prevenir su deterioro (Rowe y Kahn, 1997). En este sentido podemos hablar de capacidades de reserva internas y externas (Baltes, 1987). Las primeras se refieren al incremento del rendimiento ante tareas más complicadas como consecuencia de una práctica o ejercicio continuado. Y las segundas están relacionadas con las características del entorno que pueden ayudar a la persona a obtener mejores resultados mediante la utilización de pistas y ayudas que pueden facilitar la realización de la actividad.

Tanto en un caso como en otro las investigaciones indican que el aprendizaje durante esta etapa no solo es posible, sino altamente beneficioso para impedir o frenar el declive cognitivo (Fernández-Ballesteros, 2000; Fernández-Ballesteros, Zamarrón, Tárraga, Moya e Iñiguez 2003).

\subsection{Estimulación cognitiva y aprendizaje}

\subsubsection{Necesidad de estimulación cognitiva}

Basándose en estos principios de plasticidad neuronal, la neuropsicología ha desarrollado diferentes estrategias de estimulación cognitiva o psicoestimulación para tratar de mantener las funciones cognitivas que no han sufrido daños en la vejez y que pueden favorecer la adquisición de nuevos aprendizajes (Cullell y Vendrell, 2008; Martínez, 2002). De esta manera se pretende conseguir un envejecimiento saludable, evitar el deterioro y garantizar la independencia de la persona el mayor tiempo posible, lo que supone una mejora en su calidad de vida.

A pesar del deterioro físico y cognitivo a edades adultas, conservamos la mayoría de las células cerebrales hasta el final de nuestra vida, por lo que si seguimos utilizando nuestro cerebro de forma activa seremos capaces de construir caminos neuronales. Esta estimulación cognitiva, además de potenciar un continuo proceso creativo, ayuda a que las personas intenten esforzarse al máximo, adaptándose a su entorno y modelando su vida (Fischbach y Fischbach, 2005; Robinson y Aronia, 2004).

Fernández-Ballesteros (2006) considera que estas estrategias pueden actuar sobre varias áreas: física, mental, afectiva y social. Todas ellas desempeñan un papel importante en el proceso de envejecimiento, y la intervención en cualquiera de ellas puede contribuir a prevenir los déficits que aparecen con la edad.

Por este motivo se han desarrollado diversas actividades de psicoestimulación, como programas centrados en la memoria, talleres de educación física o relajación, de lectura o escritura, reflexoterapia, arteterapia, musicoterapia, etc.

Todas estas propuestas mejoran las capacidades cognitivas al transmitir una serie de conocimientos y fomentar la participación del individuo en actividades variadas, lo que requiere poner en marcha determinados procesos cognitivos como la atención o la memoria, entre otros (Corregidor, Moralejo y Ávila, 2007; Díaz et al, 2006). De esta manera, realizando propuestas de trabajo concretas, adaptadas a los mayores, que favorezcan su implicación y que les resulten motivadoras, estamos potenciando su funcionamiento cognitivo y previniendo en gran medida el deterioro. 
Pero estas intervenciones también tienen un fin lúdico, de diversión y socialización con otras personas, fomentando el desarrollo socio-emocional. De este modo se consigue que el anciano continúe activo a nivel físico, cognitivo, social y afectivo, mejorando su calidad de vida y promoviendo un envejecimiento saludable (Zamarrón, 2007).

Una parte importante de esta participación es la relación que se establece con su entorno. El ser humano experimenta con las cosas que le rodean a lo largo de su existencia, es la base de su vida. Por ello, necesitamos comprender que las artes son capaces de transformar la conciencia, de ayudarnos a aprender a observar el mundo (Eisner, 2004).

\subsubsection{Estimulación cognitiva: propuestas de intervención}

En Estados Unidos llevan años utilizando estas estrategias con fines terapéuticos y han obtenido buenos resultados tanto a nivel personal como en la interacción social con los demás (Ault, 1993).

Esto ha dado lugar a la aparición de diferentes propuestas terapéuticas que pretenden mejorar el funcionamiento cognitivo, afectivo y social de los mayores. Un ejemplo de ello es la musicoterapia, basada en el uso de la música para prevenir o mejorar el estado de salud física y mental (Deus, 2006). Pero también hemos de tener en cuenta la arteterapia, que utiliza la expresión artística del sujeto con un fin terapéutico, y la danzaterapia, centrada en el movimiento como medio para favorecer el desarrollo físico y personal del individuo (Di Menna, 2009). A éstas podemos añadir otras técnicas de desarrollo y motivación creativas, que fomentan un estado activo de la mente al favorecer la fluidez de ideas, sentimientos y emociones (Sbrocca y Méndez, 2007; Wortman, 2004). Al igual que el deporte favorece la tonificación de nuestros músculos, un ejercicio motivador y moderado de nuestra mente favorece el desarrollo de nuestras capacidades creativas.

Sin llegar a temas complejos, como la neurogénesis, estas técnicas y otras similares han sido utilizadas durante años tanto en personas con deterioro cognitivo o demencia como en personas sanas. El objetivo principal de todas ellas es mantener activa a la persona para evitar que se produzcan déficits asociados a la falta de participación o a la inactividad.

En los casos de deterioro físico o cognitivo estas estrategias permiten llevar a cabo un proceso de estimulación pasiva, puesto que a través de la exposición de diferentes estímulos se consigue captar la atención sensorial.

En este sentido la utilización de técnicas artísticas y creativas puede resultar sumamente interesante en este grupo de edad, dado que la actividad de nuestros sentidos no sólo es mental, sino que se inicia con la visión, el tacto, el olfato... Las artes pueden ayudar a que estas personas presten atención a lo que oyen, ven, saborean y palpan, experimentando sensaciones y haciendo que perciban cosas sin necesidad de reconocerlas, participando del poder de alfabetización visual inherente en las imágenes y obras artísticas. De esta manera la función cognitiva es un aspecto sumamente importante para el arte y la cultura. 
A través de los sentidos podemos implicar a la persona en un proceso de interacción con el mundo, aunque sea de forma pasiva, e incluso podemos conectar con su parte emocional y afectiva y retomar recuerdos y experiencias pasadas que le permitan conectar con sus seres queridos. Así podemos, de alguna manera, establecer un intercambio comunicativo y mejorar su calidad de vida (Robles, 2006; Vidret, 1996).

Pero hemos de tener en cuenta que realmente cualquier actividad creativa cotidiana, como leer, ver una película, observar una imagen o una obra artística, escuchar música, cantar, etc., repercute de forma positiva en el desarrollo, influyendo en que la persona se mantenga activa, preste atención a los estímulos que le rodean y ponga en marcha diversos procesos cognitivos (Ramírez, 1996). A partir de estas actividades también se fomenta la interacción con otros seres humanos y se mejora la autoestima, contribuyendo a prevenir el deterioro cognitivo al mantener activas las redes neuronales (García, 2008).

Y si, además, añadimos actividades constructivas, que impliquen una creación o producción propia derivada de ese intercambio artístico con el medio que le rodea, estamos involucrando al anciano en el entorno. Conseguimos mantener el contacto con el medio, fomentar la actividad y logramos que se sienta útil, aceptado y valorado por los demás. En definitiva, mantenemos sus capacidades cognitivas, afectivas y sociales, mejorando su calidad de vida (Di Menna, 2009; Sbrocca y Méndez, 2007).

En el caso de los mayores que no presentan deterioro, aparte de estas actividades, podemos plantear otras que requieran mayor implicación. Por ejemplo, elaborando creaciones artísticas de mayor complejidad, como objetos decorativos hechos con arcilla, complementos o accesorios de moda a partir de telas e hilos (bolsos, broches, collares, pulseras, etc.), elementos de cestería para usos diversos, etc. De esta forma estimulamos su mente, favoreciendo la libertad de acción y expresión, y promovemos que se sientan útiles para los que les rodean, incrementando su autoestima cuando ven que pueden generar producciones atractivas para los demás.

Pero también se pueden proponer visitas a museos o centros de arte y a conciertos o recitales para disfrutar de las exposiciones y representaciones e intercambiar opiniones sobre ellas. Con ello conseguimos nuestro objetivo de mantener las capacidades cognitivas del anciano e implicarle en el proceso de forma activa, favoreciendo la interacción con el medio. Debemos generar aquellas destrezas que favorecen el trabajo independiente y creativo de estas personas, con las siguientes finalidades:

- Fomentar la experimentación, la curiosidad y el descubrimiento.

- Estimular la expresión de ideas y sentimientos.

- Desarrollar su intuición y el proceso estético.

- Facilitar la evaluación crítica.

\section{Creatividad y desarrollo cognitivo}

La capacidad creadora es innata y no caduca, se desarrolla en torno a una flexibilidad de pensamiento o fluidez de ideas. Y es también la aptitud de concebir ideas 
nuevas o de ver nuevas relaciones entre las cosas. En algunos casos, la capacidad creadora es definida como la aptitud de pensar de forma diferente a los demás.

Por lo tanto, la creatividad, como capacidad de generar ideas o resolver problemas, se implica directamente en el desarrollo de las personas, siendo una parte importante del potencial humano, que si es promovida a lo largo de su vida sería capaz de transformar, cambiar y mejorar su existencia. En este caso, para nuestros mayores, no es tan importante la creación de nuevos productos, el planteamiento de nuevas cuestiones o la definición de nuevos problemas, sino la exploración y el descubrimiento (Gardner, 2010).

En la edad adulta, tal y como se ha justificado en el desarrollo del presente texto, este potencial se ve deteriorado en ocasiones por la falta de motivación y desarrollo, siendo necesario continuar con el proceso vital para que la persona no pierda la oportunidad de seguir expresando sentimientos, emociones y, lo más importante, ideas. Este hábito resulta muy saludable dada la pluralidad y diversidad de la sociedad actual. Los adultos, al igual que los niños necesitan tener herramientas que les permitan moverse ágilmente en un entorno plural y complejo (Robinson, 2012).

Hacer uso de las diferentes herramientas que nos proporciona el arte y la cultura, pueden favorecer con creces, el desarrollo de un aprendizaje significativo. Por todo ello, al igual que en edades tempranas, el desarrollo de un programa activo de estimulación cognitiva y creativa en personas mayores, establecería un equilibrio entre su estado madurativo y emocional.

\subsection{Motivación y desarrollo de habilidades cognitivas y creativas}

El uso del pensamiento creativo y divergente permite das respuestas alternativas, lo que permite a las personas con un estado madurativo elevado, superar obstáculos y asumir el error o la incapacidad para dar una respuesta concreta.

Un programa de estimulación y desarrollo cognitivo de nuestros mayores, basado en la motivación creativa, aportaría más "valor" a su vida. Las habilidades creativas se aprenden y se entrenan, por ello el desarrollo de un trabajo creativo, ya sea de análisis, interpretación o desarrollo gráfico, requiere la puesta en marcha de tres habilidades en el individuo (Stemberg \& O’Hara, 1999; Stemberg \& Williams, 1996):

- Habilidad analítica: relacionada con el pensamiento crítico, aunado a la capacidad que tiene el individuo de dar ideas.

- Habilidad sintética: que permite hablar sobre algo, interpretarlo o dar ideas sobre los objetos, su entorno, las personas que le rodean.

- Habilidad práctica: ligada a la abstracción y motivación personal sobre lo que hace, piensa o crea.

En este sentido, el entrenamiento creativo a edades avanzadas favorecerá su potencial de interpretación de las cosas, estableciendo una conexión e interrelación espontánea con su entorno y con las personas que le rodean.

La planificación de actividades o proyectos vinculados a las artes plásticas contribuye a la alfabetización visual. Partiendo de la simple observación de objetos po- 
demos desarrollar estas habilidades o destrezas cognitivas y emocionales. Por todo esto, es importante que estos proyectos y actividades artísticas, estén basados en generar curiosidad, lo cual les permitirá vincularse con gran sensibilidad a su entorno (Ramírez, 1996).

La motivación no está ligada a la razón ni a la mente, depende del interior del individuo, de sus emociones, de las sensaciones, de los sentimientos y de la capacidad de ser uno mismo. Por ello, podemos hacer que estas personas superen límites y barreras recobrando la confianza en ellos mismos, así como en el poder de su interioridad (Alonso, 2011; Prot, 2004).

En muchos casos, el deterioro cognitivo que presentan los mayores provoca una pérdida de la lógica, lo cual se vería subsanado por el desarrollo de un pensamiento creativo, espontáneo y divergente. En este caso, la lógica no es opuesta a la creatividad, ya que muchas actividades que están sujetas a reglas permiten crear e improvisar en su desarrollo (Robinson y Aronica, 2009).

Si conseguimos que estas personas desarrollen una "imaginación creadora" conseguiremos que sean capaces de crear o transformar algo.

\section{Conclusiones}

En los últimos cien años la esperanza de vida se ha incrementado, por eso no tenemos que cerrar puertas a los más mayores, porque la calidad de su salud también ha mejorado y debe seguir haciéndolo.

A partir de esta idea se han desarrollado diferentes propuestas de intervención sumamente interesantes para fomentar el desarrollo cognitivo durante el envejecimiento, como hemos visto en apartados anteriores. El objetivo es mantener todas sus capacidades conservadas el máximo tiempo posible para conseguir que sean miembros activos de la sociedad que les rodea, contribuyendo así a potenciar su calidad de vida.

Aunque la vejez está vinculada al paso del reloj, la edad física tiene una relación relativa con la salud y la calidad de vida. Hay personas que, con la misma edad biológica, están separadas generaciones tanto en lo emocional como en lo creativo (Fernández-Ballesteros, 2000; Lanussa, 2007). En este sentido, un trabajo artístico puede llegar a los sentimientos, al corazón y a la mente de una forma inconsciente e incluso a veces intuitiva (Robinson y Aronica, 2004).

El desarrollo y la estimulación de un pensamiento creativo adquieren una gran importancia para el ser humano, los individuos y la sociedad. Este tipo de pensamiento se considera como un comportamiento constructivo, productivo, que se manifiesta en la acción o en la realización. Por lo tanto, debe considerarse como una contribución del individuo.

Debemos diseñar programas completos que favorezcan la integración global de su interior, de sus emociones, de su mente y de sus habilidades, porque estaremos favoreciendo su autoestima, además de que se sientan realizados y, por lo tanto, más felices. Tenemos que hacer que vuelvan a ser niños, que sean curiosos, espontáneos y aventureros, que arriesguen, que sean capaces de crear, de imaginar, y que motiven sus habilidades y destrezas psicomotoras. La edad no debe influir en el impulso creativo que tenemos, ya que todos sentimos necesidad de expresar y comunicarnos. 
El pensamiento estético y la creatividad son formas de mantener activas la mente y la imaginación, y contribuyen a prevenir el deterioro cognitivo durante la vejez.

Las artes se asocian a los sentimientos, la imaginación y la autoexpresión. Por ello, aquellas personas que realicen esta práctica manual serán capaces de dar rienda suelta a numerosas ideas creativas. "El cerebro tiene en su mente mucho más que el pensamiento consciente", ya que este último solamente equivale a una pequeña parte de lo que hace en un momento determinado (Robinson, 2012). La creatividad implica "hacer algo" de forma activa y proponer actividades que necesiten el uso de algún tipo de medio: físico, como acero, madera, barro, tela o alimentos; sensorial, como el sonido, la luz, la voz o el cuerpo; sin necesidad de utilizar solamente medios cognitivos, como palabras y números.

Considerando lo comentado hasta ahora, observamos la importancia de plantear programas específicos de estimulación artística en residencias, centros de día y cualquier otro espacio compartido por personas mayores. Es importante que encontremos el medio adecuado en el que la persona se siente bien y recupera su fuerza creativa encontrándose a sí misma.

En los ancianos con deterioro estaremos estimulando sus capacidades básicas de atención y concentración, además de mejorar la comunicación con el entorno, ayudando a conectarles con sus capacidades creativas personales. Simplemente mostrándoles piezas musicales u obras de arte y proporcionándoles materiales cotidianos, como papel y pinturas o algún instrumento musical, para que expresen libremente su creatividad, estamos consiguiendo que se mantengan activos y que mejore su relación con el entorno. Es importante que mantengamos, en la medida de lo posible, la plasticidad del cerebro de un niño, capaz de absorber ideas, aprender un lenguaje, comunicarse e imaginar. La imaginación libera al ser humano de cualquier situación o circunstancia inmediata y es capaz de mantener siempre viva la posibilidad de transformar el presente (Punset, 2012; Robinson, 2012).

Estas actividades y líneas de acción, que proponemos a modo de ejemplo, no son difíciles ni costosas de llevar a la práctica, y los beneficios derivados de ellas han sido comentados ampliamente en este artículo. Consideramos que todas ellas son de gran interés y pueden obtener muy buenos resultados si se adaptan a las características personales de los participantes. Por tanto, es importante animar a las organizaciones encargadas de atender a nuestros mayores a llevarlas a cabo para conseguir un envejecimiento activo y satisfactorio, que mejorará la calidad de vida de todos los ciudadanos. Con este tipo de propuestas conseguimos mejorar la autonomía y la participación de los mayores en la sociedad, transmitiendo sus conocimientos a las nuevas generaciones. Además, logramos retrasar o, incluso, evitar el deterioro y las repercusiones sociales y económicas que éste conlleva.

\section{Referencias}

Albarracín, D., \& Berdullas, S. (2007). Psicogerontología. Infocop, 34, 3. Alonso, A. (2011). Pedagogía de la interioridad. Aprender a "ser" desde uno mismo. Madrid: Narcea. 
Ault, R. (1992). Aging artfully: health benefits of art and dance. Washington: U. S. Government Printing Office.

Ballesteros, S. (2007). Envejecimiento saludable: aspectos biológicos, psicológicos y sociales. Madrid: Universitas.

Baltes, P.B. (1987). Theoretical propositions of Life-Span Developmental Psychology: On the dynamics between growth and decline. Developmental Psychology, 23, 611-626.

Corregidor, A. I., Moralejo, C. y Ávila, M.R. (2007). Terapia ocupacional en psicogeriatría. Recuperado de http://www.terapia-ocupacional.com

Cullell, N., \& Vendrell, J.M. (2008). Aplicación de las nuevas tecnologías en los programas de intervención cognitiva para personas con deterioro. Alzheimer Real Invest Demenc., 38,14-20.

Deus, J. (2006). Estimulación cognitiva en demencias: eficacia o placebo. Informaciones Psiquiátricas, 184. Recuperado de http://www.revistahospitalarias.org

Díaz, M. C., Martín, Y., \& Peraita, H. (2006). Intervención cognitiva en personas sanas de la tercera edad (un estudio piloto en Las Rozas de Madrid). Madrid: UNED.

Di Menna, M.E. (2009). Las manifestaciones artísticas mejoran la calidad de vida en la tercera edad. Recuperado de http://www.monografias.com

Eisner, E.W. (2004). El arte y la creación de la mente. El papel de las artes visuales en la transformación de la conciencia. Barcelona: Paidos Ibérica.

European Comission (2012). ¿Cómo queremos envejecer? Recuperado de: http:// bookshop.europa.eu/es/-c-mo-queremos-envejecer--pbKE3011406/

Fairwearther, D.S. (1991). Aging as a biological phenomenon. Rev. Clinical Gerontology, 1, 3-16.

Fernández-Ballesteros, R. (2000). Gerontología Social. Madrid: Pirámide.

Fernández-Ballesteros, R., Zamarrón, M. D., Tárraga, L., Moya, R. \& Iñiguez, J. (2003). Cognitive Plasticity in Healthy, Mild cognitive Impairment (MCI) Subjects and Alzheimer's Disease Patients: A Research Project in Spain. European Psycologist, 8 (3), 148-159

Fernández-Ballesteros R. (2004). Promoción del envejecimiento activo: una perspectiva psicosocial. En L. Salvador-Carulla, J.R. Cabo-Soler y R. Cano-Sánchez (Eds.), Longevidad: tratado integral sobre salud en la segunda mitad de la vida. Madrid: Editorial Médica Panamericana.

Fernández-Ballesteros, R. (2006). Active Aging. Contribution from Psychology. En prensa.

Fernández-Ballesteros, R., Caprara, M.G., \& García, L.F. (2005). Vivir con vitalidadM: A european multimedia programme. Psychology in Spain, 9(1), 1-12.

Fischbach R.L. \& Fischbach, G.D. (2005). The brain doesn't lie. American Journal of Bioethics, 5(2), 54-55.

García, C. (2008). Sesenta y tantos. Empieza una nueva juventud. Barcelona: Ediciones CEAC.

Gardner, H. (2010). Mentes Creativas. Barcelona: Paidos Ibérica. 
Lanussa, D. G. (2007). Geriatría: conceptos generales. Recuperado de http://www. geriatricas.com

López, L. (2008). MEMORIEVOC. Programa neuropsicológico para la estimulación de la memoria. Madrid: CEPE.

Martínez, T. (2002). Estimulación cognitiva: guía y material para la intervención. Gobierno del Principado de Asturias, Consejería de Asuntos Sociales. Recuperado de http://www.imsersomayores.csic.es

Portal Mayores (2006). Psicología del envejecimiento. Recuperado de http://www. imsersomayores.csic.es

Prot, B. (2004). Pedagogía de la motivación. Cómo despertar el deseo de Aprender. Madrid: Narcea.

Punset, E. (2012). Excusas para no pensar. Cómo nos enfrentamos a las incertidumbres de nuestra vida. Barcelona: Destino.

Ramírez, M. A. (1996). Más allá de la terapia: enfoques sobre el arte en la vejez. Recuperado de http://www.redadultosmayores.com.ar/buscador/files/DESAR011. pdf

Robinson, S.K. (2012). Busca tu elemento. Aprende a ser creativo individual y colectivamente. Barcelona: Empresa Activa.

Robinson, S.K. \& Aronica, L. (2009). El Elemento. Descubrir tu pasión lo cambia todo. Barcelona: Grijalbo.

Robles, M. (2006). Arteterapia en atención geriátrica. En P. M. Domínguez (Coord.), Arteterapia. Nuevos caminos para la mejora personal y social. Sevilla: Impresores del Aljarafe.

Rowe, J.W., \& Kahn, R.L. (1997). Successful aging. The Gerontologist, 37, 433-440.

Sbrocca, F. \& Méndez, S. (2007). Talleres de arte y salud. Una propuesta para la tercera edad. $8^{\circ}$ Congreso Virtual de Psiquiatria. Interpsiquis.

Stemberg, R.J. \& O' Hara, L. (1999). Creativity and intelligence. En R.J. Stemberg (Ed.), Handbook of creativity (pp. 251-272). New York: Cambridge University Press.

Stemberg, R. J. \& Williams, W. M. (1996). How to develop student creativity. Alexandría, VA: Association for Supervision and Curriculum Development.

Tardif, S. \& Simard, M. (2011). Cognitive Stimulation Programs in Healthy Elderly: A Review. International Journal of Alzheimer's Disease, Volume 2011, 13 pages.

United Nations (2009). Population Ageing and Development. Department of economic and social affairs. Population Division. Recuperado de http://www.unpopulation.org

Vidret, M. (1996). Grieving dynamics in geriatric care: A music therapy approach. Memorias del VIII Congreso Mundial de Musicoterapia. Hamburgo, Alemania, 14-20 de julio. Hamburgo: Congress Center Hamburg: 233.

Wortman, S. (2004). Aspectos psicológicos del envejecimiento. RLG. Recuperado de http://www.gerontologia.org

Zamarrón, M.D. (2007). Envejecimiento activo. Infocop, 34, 7-9. 